\title{
O CUIDADO DE ENFERMAGEM E A ABORDAGEM WINNICOTTIANA
}

\author{
Débora Falleiros de Melloํ, Regina Aparecida Garcia de Lima²
}

\begin{abstract}
${ }^{1}$ Enfermeira. Professora Associada do Departamento de Enfermagem Materno-Infantil e de Saúde Pública da Escola de Enfermagem de Ribeirão Preto (EERP) da Universidade de São Paulo (USP). E-mail: defmello@eerp.usp.br

${ }^{2}$ Enfermeira. Professora Titular do Departamento de Enfermagem Materno-Infantil e de Saúde Pública da EERP/USP. E-mail: limare@eerp.usp.br
\end{abstract}

RESUMO: Este estudo tem por objetivo identificar contribuições da abordagem teórica winnicotianna ao cuidado de enfermagem. Estudo reflexivo que destaca os conceitos holding, ambiente facilitador, espaço transicional e relações suficientemente boas e estabelece interface com o cuidado em saúde, relevantes para ampliar a compreensão do cuidado de enfermagem. O cuidado de enfermagem é tomado na perspectiva das práticas suficientemente boas, requerendo a construção de espaços de mutualidade, suporte, proteção e ambiente facilitador. Os conceitos winnicottianos agregam novos valores ao cuidado de enfermagem, permitindo uma vitalidade conceitual para o cuidado numa perspectiva integradora.

DESCRITORES: Enfermagem. Cuidado em saúde. Psicanálise.

\section{NURSING CARE AND THE WINNICOTT APPROACH}

\begin{abstract}
This study aimed to identify contributions of Winnicott's theoretical approach to nursing care. It is a reflexive study that highlights the concepts of holding, facilitating environment, and transitional space. It establishes interface with health care, relevant for nursing care comprehension. Nursing care is approached in the perspective of "good enough" practices, requiring the establishment of arenas for mutual contact, support, protection, and facilitating environment. Winnicott concepts add new values to nursing care, enabling a conceptual vitality for care in a comprehensive perspective.
\end{abstract}

DESCRIPTORS: Nursing. Health care. Psychoanalysis.

\section{EL CUIDADO DE ENFERMERÍA Y LA APROXIMACIÓN TEÓRICA WINNICOTTIANA}

\begin{abstract}
RESUMEN: El objetivo de este estudio es identificar las contribuciones de la aproximación teórica winnicottianna al cuidado de enfermería. Es un estudio reflexivo en el que se destacan los siguientes conceptos: holding, ambiente facilitador y espacio transicional, y se establece interfaz con el cuidado en salud, importantes conceptos para la comprensión del cuidado de enfermería. El cuidado de enfermería es enfocado desde la perspectiva de las prácticas suficientemente buenas, requiriendo la construcción de espacios de mutualidad, apoyo, protección y entorno propicio. Los conceptos winnicottianos agregan nuevos valores a la atención de enfermería, permitiendo una vitalidad conceptual para el cuidado en una perspectiva integradora
\end{abstract}

DESCRIPTORES: Enfermería. Cuidado en salud. Psicoanálisis. 


\section{INTRODUÇÃO}

Atualmente, as práticas de saúde vêm sendo discutidas no que se refere a um expressivo desenvolvimento científico e tecnológico, e suas limitações, para responder efetivamente às complexas necessidades de saúde de indivíduos e populações, assim como temas sobre humanização da atenção à saúde, integralidade do cuidado à saúde, promoção da saúde, qualidade, equidade, autonomia e direitos dos usuários têm se configurado em estratégias e princípios para a organização das práticas de atenção à saúde. ${ }^{1-4}$

É relevante retomar os conceitos que envolvem o cuidado em saúde, repensando o encontro terapêutico, que não deve ser movido exclusivamente pela técnica, mas com o desafio ético de não deixar a técnica substituir o encontro, buscando a qualidade desse encontro. $^{5}$

A enfermagem é um grupo profissional de singular importância, pelo desempenho de papéis, funções e responsabilidades, que são específicas do cuidado à saúde. A essência da profissão enfermagem é o cuidado ao ser humano, seja individualmente, na família ou na comunidade, para o desenvolvimento de intervenções de promoção da saúde, na prevenção de doenças, e de recuperação e reabilitação da saúde. ${ }^{6}$

No presente estudo, o cuidado de enfermagem está focado no encontro com o outro, a partir da compreensão do cuidado em saúde e suas interfaces com conceitos winnicottianos, justificando que tais conceitos trazem elementos importantes para o entendimento do cuidado, sendo interessante explorá-los no âmbito da enfermagem. Os estudos de D. W. Winnicott exploram um modo particular de pensar as primeiras relações da vida do ser humano e sua constituição vinculada aos cuidados maternos. Esse pensamento traz contribuições aos profissionais de saúde nas dimensões da prática assistencial, pesquisa e ensino, com ênfase no cuidado. Os conceitos elaborados por esse autor sobre holding, ambiente facilitador, espaço transicional e relações suficientemente boas exploram a importância da pessoa e do ambiente para o desenvolvimento do indivíduo. ${ }^{7-9}$ Tais conceitos têm aproximação com os aspectos de intersubjetividade e de construção da relação com o outro, fundamental para ampliar a compreensão do cuidado de enfermagem.

Poucos estudos na área de enfermagem têm empregado os conceitos winnicottianos. Um estudo abordou como a supervisão clínica de enfermagem pode ser enriquecida a partir da utilização dos conceitos de holding e apresentação de objetos. Nele, a autora demonstrou o potencial desses conceitos, apontando que holding pode ser entendido como a habilidade para empatia, e que, na supervisão clínica, é importante a criação de um ambiente facilitador e o estabelecimento de um relacionamento que inclua o manejo e a apresentação de objetos, criando condições para o vínculo e a segurança profissional, com mudanças e adaptações possíveis e fazendo com que o supervisionando seja capaz de acreditar que ele possa ser o autor de sua prática. ${ }^{10}$

O trabalho em equipe também já foi analisado à luz da teoria winnicottiana, apontando que a equipe de profissionais de saúde tem o papel de cuidar, e que ela pode ser cuidadora de si, desde que experimente, no seu interior, uma dinâmica de relacionamento, em direção a um bom holding entre os profissionais de saúde. ${ }^{11}$

O conceito winnicottianno de holding também foi estudado no cuidado de enfermagem a idosos, ilustrando que o desenvolvimento de habilidades de interação com esses pacientes tornase ampliado a partir desse conceito, permitindo compreensão da interação social e incremento da autoestima dos pacientes. ${ }^{12}$

Um outro estudo sobre cuidados com bebês em maternidade, verificou o holding proporcionado por enfermeiras, mães e pais, aos bebês hospitalizados em uma unidade neonatal de médio risco. Observou-se, por meio de filmagens, que alguns profissionais falharam no fornecimento do holding, enquanto outros proporcionaram um holding satisfatório, trazendo para discussão que a prática de enfermagem pode ser aperfeiçoada de modo que todos os membros da equipe, assim como as mães e os pais sejam capazes de fornecer o holding adequado, visando um bom desenvolvimento infantil. ${ }^{13}$

A assistência à saúde da criança, a partir de relatos de mães e de profissionais na atenção primária à saúde, também foi estudada estabelecendo uma aproximação com a abordagem winnicottiana, destacando a importância da preocupação que os profissionais de saúde devem ter com os valores que as mães e as famílias dão para o cuidado cotidiano da criança, evidenciando a ênfase que a referida abordagem dá aos papéis de enfermeiros e médicos na construção da segurança e confiança materna e familiar. ${ }^{14}$

$O$ cuidado de enfermagem, principalmente no que diz respeito ao cuidado enquanto preocupação com o outro, pode ser mais explorado. 
Nesse sentido, considera-se que os conceitos winnicottianos podem conter ricas contribuições ao entendimento das relações entre profissionais de saúde e pacientes. Dessa forma, a apreensão de alguns conceitos permite a construção de uma reflexão teórica, expressando relações entre dois ou mais conceitos, de forma útil e eficiente. ${ }^{15}$

Este estudo reflexivo busca apresentar alguns traços dos conceitos winnicottianos para ampliar a compreensão do cuidado de enfermagem. O objetivo é identificar contribuições da abordagem teórica winnicotianna ao cuidado de enfermagem, com vistas a agregar novos valores à prática e à pesquisa em enfermagem.

\section{CUIDADO E PREOCUPAÇÃO COM O OUTRO NA PERSPECTIVA WINNICOT- TIANA}

A abordagem winnicottiana tem como foco o relacionamento mãe-bebê. Em suas primeiras fases, o bebê passa por um processo de identificação primária e a mãe representa o ambiente primário. ${ }^{9} \mathrm{O}$ bebê é dependente e tem poucas condições simbólicas e possibilidades de representação, sendo fundamental a função de continência, ou seja, que tenha condições de suportar, de dar conta de, de não se descontrolar. Assim, os pais precisam de condições para promover o desenvolvimento dos filhos.

Mãe e bebê têm uma experiência de mutualidade e a preocupação (concern) materna primária é um conceito utilizado na abordagem winnicottiana para descrever o estado psicológico especial da mãe, durante os primeiros tempos da criação do bebê, particularmente os cuidados corporais e a elaboração da relação com o filho. A mãe vai se identificando e dando sustentação física e emocional nesta etapa de dependência absoluta do bebê.

O conceito winnicottiano de holding é descrito como uma fase em que a mãe ou substituta protege o bebê de agressões, leva em conta a sensibilidade cutânea, inclui uma rotina de cuidados diurnos e noturnos e observa mudanças físicas e psicológicas do cotidiano. ${ }^{7-9} \mathrm{O}$ holding está ligado à capacidade da mãe de identificar-se com o seu bebê e um holding construído de forma satisfatória é considerado uma porção básica de cuidado. Um holding deficiente produz extrema insegurança na criança, sendo associado a sensações de despedaçamento, desligamento, sentimentos de que a realidade exterior não reconforta o mundo interior. ${ }^{9}$
O holding é construído pelo suporte materno, em suas funções de sustentação, proteção, reconhecimento, aceitação, adaptação ao ritmo único e original de cada criança e acompanhamento de mudanças sutis. Faz parte da constituição das relações no processo de crescimento e desenvolvimento infantil, imprimindo uma adaptação viva às necessidades do bebê. Essa adaptação dinâmica define a função da mãe suficientemente boa., ${ }^{716}$

Mãe suficientemente boa é outro conceito winnicottiano que está ligado ao processo das relações suficientemente boas de proporcionar condições ao bebê para tolerar frustrações e conflitos com a realidade, aprender a aguardar e adiar, mediando o que é suportável para a criança. Este conceito está vinculado a outro, denominado ambiente facilitador. ${ }^{8}$

O conceito de ambiente facilitador diz respeito às condições fornecidas por um ambiente adequado que se adapte às necessidades do bebê. Na perspectiva winnicottiana o ambiente descrito não é interno nem mesmo externo, mas refere-se a uma instância que sustenta e responde à dependência, ou seja, que o bebê necessita totalmente de um outro que ainda não é um outro, separado ou externo a ele. A realidade de si mesmo e a realidade do mundo são construídas durante o processo de amadurecimento, no interior da relação com o cuidador.

O processo maturacional do bebê impulsiona o relacionamento com a mãe e com o pai, com o elemento humano, sendo apontado que "[...] uma grande parte da vida saudável tem a ver com as várias modalidades de relacionamento objetal e com um processo de 'vaivém' entre o relacionamento com objetos externos e internos. Isso é uma questão de pleno usufruto das relações interpessoais" . 8:13

A partir das percepções dos objetos e das relações entre o mundo interno e o mundo externo há uma importante área de experiência, que configura o espaço potencial existente entre o indivíduo e o meio ambiente. Esse espaço, também chamado de área intermediária e espaço transicional, é outro conceito winnicottiano tratado pelo autor, para falar de objetos e fenômenos transicionais como fonte de experiências culturais. ${ }^{7-9}$

O termo objeto transicional foi criado pelo autor para retratar o uso de certos objetos (ursinho, paninho, cobertor, chupeta, entre outros) pela criança, dando expressão para a área intermediária entre o subjetivo e o objetivo. No processo de crescimento e desenvolvimento do bebê, o surgimento do objeto transicional está relacionado à conforma- 
ção do vínculo mãe-filho. A utilização de objetos transicionais possibilita criar suportes que vão permitindo ao bebê ultrapassar fases, continuar o caminho em direção à mãe e à satisfação ou então esperar o regresso dela, sem se desesperar. ${ }^{16}$

$O$ objeto transicional tem um significado próprio para o bebê que, ao mesmo tempo, cria e imagina, o que permite a construção de algo que é "não eu" e que pode se relacionar com ele. ${ }^{9}$ Este objeto é muito importante para o bebê e é usado em momentos de dormir, de solidão e ansiedade, ou para confortar-se, sendo difícil separar-se dele. Com base em um desenvolvimento saudável, com o passar do tempo, o objeto deixa de ser usado ou é esquecido.

A manipulação do objeto é acompanhada de uma atividade de fantasias, observada pelo autor como área de ilusão. ${ }^{9} \mathrm{O}$ objeto representa a mãe e também algo do mundo interno do bebê. Com o passar do tempo, ele vai percebendo e construindo as diferenças, as realidades interna e externa, embora inter-relacionadas, vão se distinguindo, ou seja, vai se processando gradativamente a desilusão. $O$ objeto transicional possibilita um processo de transição na relação mãe-bebê,, modificando a relação de um tempo de identificação, de indiferenciação e de ilusão, para um tempo de separação e constituição do eu. Assim, o autor estuda a experiência ilusória em um percurso que vai da ilusão individual aos fenômenos culturais, criando uma trama para a vida, afirmando que todo esse processo constitui uma proteção contra consolidações patológicas. Para o autor, os fenômenos transicionais conduzem à criatividade, às mudanças, à liberdade individual e aos potenciais da civilização. ${ }^{7-9}$

Na perspectiva winnicottiana, a criatividade de um indivíduo está muito relacionada com sua capacidade simbólica, originada pelo processo de ampliação dos fenômenos transicionais. Os fenômenos transicionais são essenciais para o intercâmbio entre o bebê e o mundo. A criança vai crescendo e conseguindo realizar abstrações. No adulto, esse processo de inter-relações é representado pela imaginação, pelo trabalho criativo, pelas artes e pela religião. O espaço transicional é considerado o patrimônio de cada indivíduo. ${ }^{16}$

Todos esses conceitos winnicottianos são interessantes para pensar o cuidado em saúde, a fim de discutir o encontro e a interação entre os sujeitos, assim como as possibilidades de reconstrução das práticas de saúde, a partir da reflexão dos modos de nos relacionarmos com os outros.

\section{A ENFERMAGEM E O CUIDADO SUFI- CIENTEMENTE BOM}

O profissional enfermeiro tem uma posição chave para o desenvolvimento e utilização de estratégias inovadoras na promoção da saúde, na prevenção de doenças, e na manutenção e recuperação da saúde, de forma indissociável. Nesse sentido, a perspectiva do cuidado integral à saúde implica repensar as formas de interação com os sujeitos, seja no processo saúde-doença ou no cuidado.

O cuidado de enfermagem é entendido como um ato comunicativo que requer conhecimentos específicos e de compreensão do contexto onde vivem, trabalham e adoecem as pessoas. $\mathrm{O}$ desenvolvimento das competências de enfermagem, que vai além da aquisição de conhecimentos teóricos e técnicos, necessita também da apropriação e desenvolvimento de atitudes para melhorar a qualidade da relação interpessoal e da comunicação para o cuidado. ${ }^{17}$

Estabelecendo uma aproximação aos conceitos winnicottianos aqui destacados, na particularidade da comunicação e interação com o outro, faz-se importante considerar que o cuidado de enfermagem é permeado por um processo de identificação com o sujeito que está sendo cuidado. $\mathrm{O}$ profissional enfermeiro faz parte da construção de identidade do sujeito, nos aspectos de sua saúde e, de como este cuida da sua saúde e compreende seu processo saúde-doença, bem como o que significa o ambiente de cuidado e as condições de saúde. O cuidado em saúde envolve voltar-se à presença do outro, otimizar a interação e enriquecer os horizontes com interações intersubjetivas ricas e dinâmicas. ${ }^{1,18}$

No processo de cuidar, e nessa construção da identidade de cuidador, é importante que o profissional de enfermagem construa a função de continência e holding, tomando por base a abordagem winnicottiana. Esse processo está vinculado a um movimento de compartilhar, dar suporte, proteção e sustentação, demonstrar preocupação e compreensão da situação de saúde e de vida dos sujeitos.

O cuidado de enfermagem tem proximidade ao conceito winnicottiano de holding 9 e à sua continuidade na vida adulta, pela necessidade de proteção do indivíduo contra riscos, danos e agravos à saúde, levando em conta a sensibilidade e a observação de mudanças físicas e psicológicas e de repercussões que a enfermidade pode trazer ao co- 
tidiano. O holding pode estar ligado à capacidade do enfermeiro de identificar-se com o sujeito que está sendo cuidado e com suas necessidades de saúde contextualizadas, para evitar distanciamento, desligamento ou não adesão ao tratamento, e sentimentos de que o profissional de saúde não o reconforta. Nesse sentido, a construção de compartilhamento e suporte caminha junto do diálogo, da conversa e da fusão de horizontes, para o desenvolvimento da pessoa e do cuidado de sua saúde.

$\mathrm{Na}$ interação com o sujeito, o cuidado de enfermagem requer a construção de espaços de mutualidade e um ambiente facilitador. O conceito winnicottiano de ambiente facilitador ${ }^{9}$ diz respeito às condições fornecidas por um ambiente adequado, que proporcione adaptação às necessidades dos sujeitos envolvidos. Construir um ambiente facilitador pode permitir que experiências de mutualidade e de interação construam a conexão com o outro, com a situação de adoecimento, sofrimento e tratamento. $\mathrm{O}$ ambiente que a enfermagem proporciona aos sujeitos pode ou não ser propício à identificação e ao alcance das necessidades de saúde individuais e coletivas, às intervenções de promoção, prevenção, terapêutica e reabilitação de forma indissociável, ao movimento de autonomia para o cuidado, entre outros aspectos. Um ambiente que proporcione cuidado é acolhedor, tolerante, seguro, confiável e protetor. ${ }^{19}$

Cabe também repensar o cuidado de enfermagem a partir do entendimento do conceito winnicottiano de espaço transicional ${ }^{9}$, visando mudanças, liberdade e criatividade. O profissional de enfermagem pode compreender e expandir espaços transicionais e possibilitar que as experiências, as idéias e os pensamentos dos sujeitos façam parte do plano de cuidados e da conformação de vínculos. No processo de cuidar é importante estar atento à modificação da relação com o outro, de um tempo de identificação das necessidades de saúde para um tempo de constituição da autonomia, em busca de um entendimento ampliado do processo saúde-doença. O cuidado de enfermagem, ao privilegiar a preocupação com o amadurecimento do sujeito que é cuidado, pode construir um processo que permite o crescimento pessoal, o pleno benefício das relações interpessoais e a autonomia, buscando um sentido de responsabilidade, de tomar para si, de responsabilizar-se e de estabelecer uma efetiva interação na perspectiva do cuidado integral à saúde.

O conceito winnicottiano de mãe suficientemente boa ${ }^{9}$ pode contribuir para ampliar a visão do cuidado de enfermagem, na medida em que revigora as relações suficientemente boas, com processos de mediação entre tolerância, suporte, frustração e compreensão de conflitos. Em geral, nos processos saúde-doença, os sujeitos sentem e se preocupam com sua saúde, mas vão até certo ponto e depois precisam do outro e da relação com os serviços de saúde. Eles têm necessidades de orientação, apoio e tratamento para realizar os cuidados que normalmente realizam, mas há limites. Nesse processo, os sujeitos experimentam os limites das insuficiências e, em vários momentos, a busca da suficiência vai ocorrer nos serviços de saúde.

Tomar o cuidado de enfermagem como um processo vivo e suficientemente bom implica lidar com diferentes condições de orientação, aceitação, conflito e frustração, que necessitam tolerância, compreensão, adaptação dinâmica, suporte e mediação. Desse modo, é de extrema importância tematizar com os sujeitos, aspectos de seu interesse, repensar sobre aquilo que os mobiliza e os desacomoda, estimular a produção de narrativas terapêuticas, conhecer as escolhas e tomadas de decisão, fortalecer virtudes e experiências cotidianas positivas, contribuindo para ampliação desse cuidado, no sentido de apreendê-lo sob uma perspectiva integradora e suficientemente boa.

Assim, é importante apreender o cuidado de enfermagem na perspectiva das práticas suficientemente boas. Trata-se de um deslocamento discursivo do conceito de "mãe suficientemente boa" para a idéia de "cuidado suficientemente bom".

Os conceitos winnicottianos evidenciam a importância da interação para o desenvolvimento do ser humano $\mathrm{o}^{7-9} \mathrm{e}$, nesse aspecto, está diretamente vinculado ao entendimento do cuidado em saúde e, particularmente, do cuidado de enfermagem.

O cuidado em saúde deve considerar a intersubjetividade em uma relação que deve ser entendida no sentido de encontro, que denota o ato de se colocar diante do outro, significando compossibilidades e compartilhamentos. ${ }^{2}$

As diferentes situações de vulnerabilidade por que passam os sujeitos precisam ser particularizadas nas práticas de saúde, qualquer que seja a fragilidade ou o dano, requerendo atenção do profissional de enfermagem para estar junto, seja no processo saúde-doença e no cuidado.

O entendimento é que o cuidado de enfermagem é essencialmente relacional e contingencial. A reflexão sobre esse caráter relacional e contingente de nossas identidades e historicidades como in- 
divíduos e grupos é de fundamental importância porque são elementos intrínsecos do cuidar. ${ }^{2,6,18}$ $\mathrm{O}$ cuidado tem caráter contingencial porque implica em lidar com situações imprevisíveis, com a eventualidade, a incerteza e as diferenças dos acontecimentos vinculados às experiências. ${ }^{14}$

Tomar o cuidado de enfermagem com essas particularidades significa necessariamente integrar saberes práticos e saberes técnicos, com efetiva articulação entre os conhecimentos técnicocientíficos e as experiências dos sujeitos.

As experiências dos sujeitos estão no âmbito da sabedoria prática, a qual é apontada como o diferencial que torna possível o movimento de humanização do encontro entre sujeitos e sua transformação em cuidado. ${ }^{6}$ Sabedoria prática é um conceito originário da filosofia prática, de modo contingente, que não lida com aspectos perenes, causais e universais, tampouco, é um saber que produz objetos, artefatos ou instrumentos. ${ }^{20}$ É um conhecimento que emerge das experiências, dos interesses comuns ou divergentes, de tensões e de possibilidades de interação. Por se tratar de um espaço para enfocar os interesses humanos, não há grau de menor verdade, mas sim, menos certeza e determinação. A preocupação, portanto, é com a construção da busca de compreensão da vida, das experiências e escolhas diante das diversas possibilidades no cotidiano. ${ }^{20}$

O conhecimento de enfermagem pode ser enriquecido com o aprofundamento de estudos e discussões sobre os conceitos winnicottianos e sobre o conceito de sabedoria prática.

\section{CONSIDERAÇÕES FINAIS}

Os conceitos winnicottianos de holding, ambiente facilitador, espaço transicional e relações suficientemente boas agregam novos valores ao cuidado de enfermagem, permitindo uma vitalidade conceitual para o cuidado numa perspectiva integradora.

O cuidado de enfermagem não se dirige somente a um processo saúde-doença linear, pois, há obstáculos, valores e percepções da enfermidade e do adoecimento, situações e experiências de cuidar que se expressam de várias maneiras. É importante a construção de horizontes, em conjunto com os sujeitos que estão sendo cuidados, revendo a sua saúde. Se faz pertinente uma atitude profissional reconstrutiva para que as pessoas possam buscar e se apropriar de informações e conhecimentos sobre saúde, que façam sentido para elas. Desse modo, é de extrema importância ouvir o outro, compreender e discordar, entender e concordar o que é colocado de verdade para o outro e o que o outro coloca, não com a idéia de um horizonte final a ser alcançado, mas sim um horizonte possível e processual, revendo e repensando os obstáculos na interação, de maneira reconstrutiva e suficientemente boa.

No presente estudo, buscou-se uma aproximação a alguns conceitos winnicottianos, com uma síntese de seus traços constitutivos, tomando como foco o cuidado de enfermagem. Os conceitos aqui destacados não apresentam uma aplicabilidade imediata, mas oferecem possibilidades de abstração e um entendimento do cuidado. Cabe ressaltar que outros espaços de discussão e de pesquisas poderão configurar a densidade desses conceitos em diferentes áreas da saúde e expandir as reflexões em outras dimensões epistemológicas e práticas.

\section{REFERÊNCIAS}

1. Ayres JRCM. O cuidado, os modos de ser (do) humano e as práticas de saúde. Saúde Soc. 2004 Set-Dez; 13(3):6-29.

2. Ayres JRCM. Sujeito, intersubjetividade e práticas de saúde. Ciênc Saúde Coletiva. 2001 Jan-Fev; 6(1):63-72.

3. Costa AM. Integralidade na atenção e no cuidado à saúde. Saúde Soc. 2004 Set-Dez; 13(3):5-15.

4. Fortes PAC. Ética, direitos dos usuários e políticas de humanização da atenção à saúde. Saúde Soc. 2004 Set-Dez; 13(3):30-5.

5. Ayres JRCM. Cuidado e humanização das práticas de saúde. In: Deslandes S, organizadora. Humanização dos cuidados em saúde: conceitos, dilemas e práticas. Rio de Janeiro (RJ): Fiocruz; 2006. p.49-83.

6. Rocha SMM, Almeida MCP. O processo de trabalho da enfermagem em saúde coletiva e a interdisciplinaridade. Rev Latino-am Enferm. 2000 Dez; 8(6):96-101.

7. Winncott DW. O ambiente e os processos de maturação: estudos sobrea teoria do desenvolvimento emocional. Porto Alegre (RS): Artes Médicas; 1988.

8. Winncott DW. Tudo começa em casa. São Paulo (SP): Martins Fontes; 1999.

9. Winncott DW. A família e o desenvolvimento individual. São Paulo (SP): Martins Fontes; 2005.

10. Rafferty MA. A conceptual model for clinical supervision in nursing and health visiting based upon Winnicott's (1960) theory of the parent-infant relationship. J Psychiatr Ment Health Nurs. 2000 Apr; 7(1):153-61.

11. Campos EP. Equipe de saúde: cuidadores sob tensão. Epistemo-somática, 2006 Set-Dez; 3(2):195-222. 
12. McGilton KS. Enhancing relationships between care providers and residents in long-term care: designing a model care. J Gerontol Nurs. 2002 Dec; 28(12):13-21.

13. Regis F C, Kakehashi T Y, Pinheiro E M. Análise do cuidado ao bebê hospitalizado segundo a perspectiva winnicottiana. Rev Bras Enferm. 2005 Jan-Fev; 58(1):39-43.

14. Mello DF, Lima RAG, Scochi CGS. Health follow-up of children in poverty situation: between the routine and eventuality of daily care. Rev Latino-am Enferm. 2007 Set-Out; 15(spe):820-7.

15. Walker LO, Avant KC. Strategies for theory construction in nursing. New Jersey (US): Pearson Prentice Hall; 2004.
16. Abadi S. Transições: o modelo terapêutico de D.W. Winnicott. São Paulo (SP): Casa do Psicólogo; 1998.

17. Bermejo JC. Salir de la noche: por uma enfermería humanizada. Serie: Humanizar la salud. Santander (España): Editorial Salterrae; 1999.

18. Ayres JRCM. Ayres JRCM. Hermenêutica e humanização das práticas de saúde. Ciênc Saúde Coletiva. 2005 Jul-Set; 10(3):549-60.

19. Araújo MI, Galiza W, Ângelo AC. O gesto espontâneo e o meio ambiente na perspectiva winnicottiana. Rev Conceitos. 2001 Jan-Dez; 4(5):80-3.

20. Ayres JRCM. Para comprender el sentido práctico de las acciones de salud: contribuciones de la Hermenéutica Filosófica. Salud Colectiva. 2008 May-Ago; 4(2):159-72.

E-mail: defmello@eerp.usp.br 